\title{
Evolution and Fragmentation of Wide-Angle Wind Driven Molecular Outflows
}

\author{
Andrew Cunningham, Adam Frank, Peggy Varnière and \\ Alexei Poludnenko \\ University of Rochester \\ Sorin Mitran \\ University of North Carolina, Chapel Hill \\ Lee Hartmann \\ Harvard-Smithsonian Center for Astrophysics
}

\begin{abstract}
We present two dimensional cylindrically symmetric hydrodynamic simulations and synthetic emission maps of a stellar wind propagating into an infalling, rotating environment. The resulting outflow morphology, collimation and stability observed in these simulations have relevance to the study of young stellar objects, Herbig-Haro jets and molecular outflows. Our code follows hydrogen gas with molecular, atomic and ionic components tracking the associated time dependent molecular chemistry and ionization dynamics with radiative cooling appropriate for a dense molecular gas. We present tests of the code as well as new simulations which indicate the presence of instabilities in the wind-blown bubble's swept-up shell.
\end{abstract}

Keywords: Protostellar Outflow, HH Object, Molecular Outflow, Wide-Angle Wind, Fragmentation

\section{Introduction}

Bipolar Jets and wide angle molecular outflows are recognized as a ubiquitous phenomena associated with star formation. It is expected that most if not all low mass stars produce such outflows during their formation through the gravitational collapse of gas from the parent molecular cloud. A molecular outflow is formed when molecular gas is displaced from the cavity evacuated by a fast stellar wind. This results in the formation of irregular lobes and thin shells of swept up shocked molecular gas along the walls of the cavity. The strong radiative energy loss from the shock heated molecular gas can result in the onset of several instabilities in the molecular outflow (Vishniac, 1994), (Vishniac \& Ryu, 1989). We present some preliminary results of our work employing multidimensional numerical models including molecular chemistry and associated radiative losses to explore the fragmentation and stability properties of these outflows.

Simulations of protostellar outflows in the presence of a collapsing molecular core have been carried out using the AstroBEAR adaptive

(C) 2018 Kluwer Academic Publishers. Printed in the Netherlands. 
mesh refinement muti-physics code (Poludnenko et. al., 2004), (Varnie et. al., 2004). The AstroBEAR code employs an exact hydrodynamic Riemann solver and a conservative integration scheme in an Eulerian frame of reference to advance the solution of the source free Euler equations. The geometric and microphysical source terms are split from the hydrodynamic integration using an implicit fourth-order Rosenbrock source term integration scheme for stiff ODE's. The use of adaptive mesh code has been essential to achieve the necessary resolution in the neighborhood of thin shock bounded high density slabs that are prevalent in these simulations.

\section{Isothermal X-wind Model Simulation}

We first compare our code with results of previous calculations. In figure 1 we show a simulation of an isothermal outflow with conditions similar to the X-wind model of Shu et. al. (1995) driving into a toroidal ambient medium. This simulation uses a velocity and density pattern similar to the $\mathrm{X}$-wind but no magnetic field is included. We compare figure 1 with the results of the same calculation performed by Lee et. al. (2001) at 1/4 the resolution of the current work. Of particular interest is the extent to which the late-time flow morphology depicted in figure 1 agrees with the analytically predicted result of Lee et. al. (2001) delineated by the black curve in the figures as a verification of our code in these regimes.

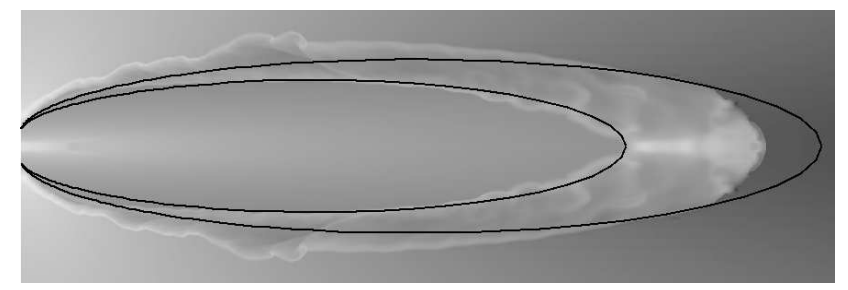

Figure 1. X-wind outflow at $\mathrm{t}=304 \mathrm{yr}$. The outer curve represents the analytically predicted shell morphology at this time. The inner curve follows the shape of the analytical model at an earlier time and whose morphology matches the shape of the inner shock in our simulations.

The higher resolution in our simulation allows us to track the internal dynamics of the swept-up shell. Our simulations reveals fine scale features generated as the material flows along the shell walls something not possible with the lower resolution study. Note that the analytic shell morphology model assumes a thin shell. The violation of this conditions is likely responsible for the deviation from the analytically predicted 
morphology given by the outer curve. The general form of the outflow morphology is, however, well approximated by the analytic model.

\section{Protostellar Wind-Infall Model with Non-Equilibrium Ionization, $\mathrm{H}_{2}$ Chemistry and Cooling}

We have also performed simulations including the effects of non-equilibrium HI ionization (Arnaud \& Rothenflug, 1985), (Hollenbach \& McKee, 1977), (Mazzotta et. al., 1998), H2 dissociation and cooling (Dove \& Mandy, 1986), (Lepp \& Schull, 1983), (Lim, et. al., 2002), (Mandy \& Martin, 1993), (Martin et. al., 1998). OI line cooling, a dominant cooling agent at temperatures below $1000 \mathrm{~K}$ has also been included (Launay \& Roueff, 1977). Atomic line and recombination cooling has been included using the cooling rates of a coronal gas Dalgarno \& McCray (1972). The volumetric cooling rates for the case of a partially ionized gas are plotted in figure 2. We have also performed tests of our chemistry routines. We show one of these tests where we find the shock speeds which produce $90 \%$ molecular dissociation. Figure 3) shows our code produces results consistent with those of previous authors (Smith, 1994), (Hollenbach \& McKee, 1977).

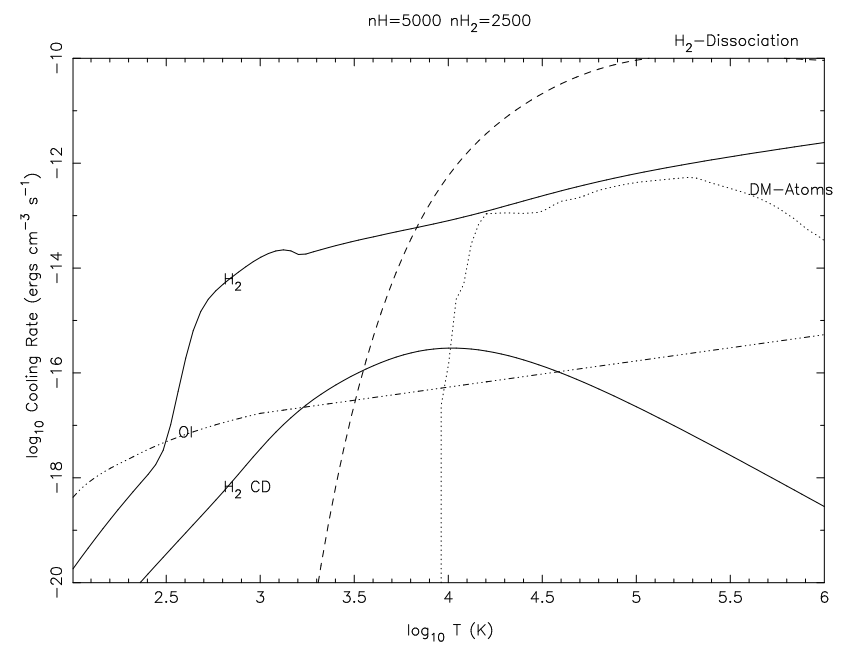

Figure 2. Cooling rates for typical ISM abundances, $n_{H_{2}}=2500, n_{H I}=5000 . H_{2}$ is the molecular hydrogen cooling function for a tenuous gas, $\mathrm{H}_{2} \mathrm{CD}$ is the molecular cooling function valid for $n>>n_{\text {critical }}, H 2-$ Dissociation is the thermal energy loss due to the dissociation of $\mathrm{H}_{2}$ molecules, $\mathrm{OI}$ is the singly ionized oxygen line cooling and $D M-$ atoms is the atomic line and recombination cooling function of Dalgarno \& McCray. 


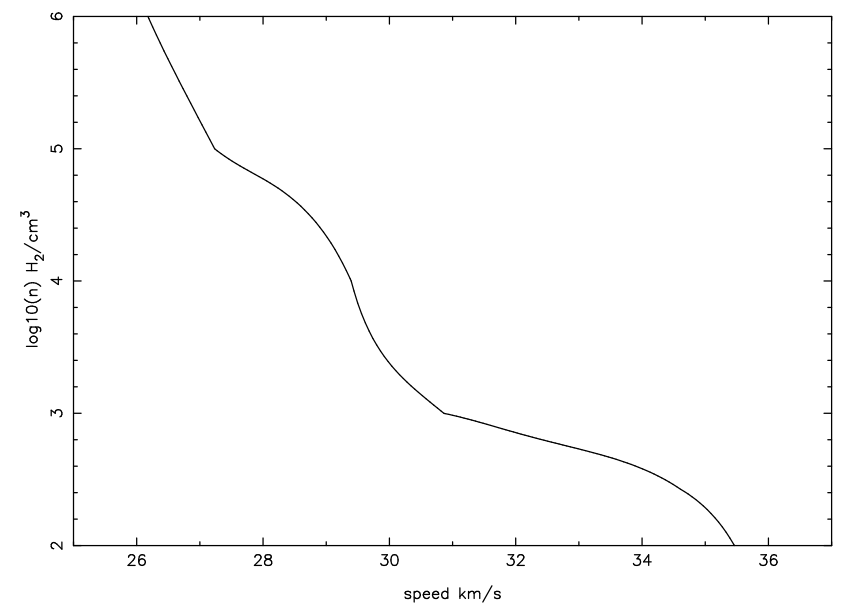

Figure 3. Preshock density vs. shock speed for a steady shock resulting in $90 \%$ downstream $\mathrm{H}_{2}$ dissociation.

Figure 4 shows the interaction of a tenuous, initially ionized protostellar wind with a cold, slowly rotating molecular environment in the presence of the central gravitational potential of .21 $M_{\odot}$ protostar (not resolved in these simulations). The model for the molecular environment is that of a collapsing of non-magnetic, self-gravitating sheet of Hartmann et. al. (1996) as implemented by Delamarter et. al. (2000). The initial outflow takes the form of a sphere ejecting gas at a uniform velocity with an azimuthal density gradient varying as $\cos (\theta)$ and an equator to pole density contrast of 50 . Thus our inflow condition creates a wide angle wind in which the momentum input to the environment by the protostar is aspherical with the bulk of the thrust being directed along the poles.

The outflow speed in the simulation is maintained at $100 \mathrm{~km} / \mathrm{s}$ with the inflow injected through a "wind sphere" of radius of $33 \mathrm{AU}$, an outflow rate of $10^{-8} M_{\odot} y r^{-1}$ and an initial infall rate of $10^{-9} M_{\odot} y r^{-1}$ from the collapsing environment. The equatorial outflow ram pressure is overcome by the gravitational infall of the shocked ambient gas resulting in appreciable shock focusing of the outflow. This leads to very efficient collimation of the ejected wind material.

The resolution achieved in these simulations is sufficient to resolve the swept up shells to 6 to 10 pixels. This is enough to track the onset of what appear to be thin shell instabilities. The shell fragmentation process resulting from such instabilities may have a significant effect on the efficiency with which the outflow is able to entrain and disperse 


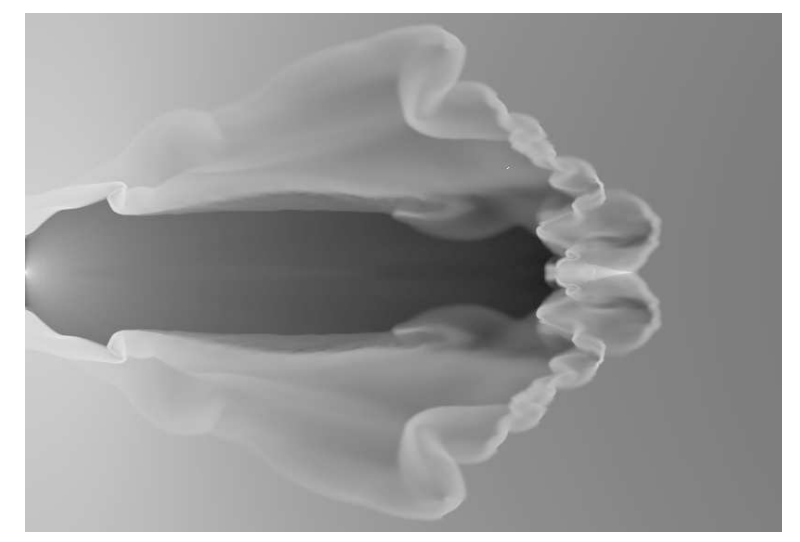

Figure 4. Collapsing sheet outflow, low speed case, logarithm of density at $\mathrm{t}=97 \mathrm{yr}$.

its momentum in support of the collapsing environment and on the morphological signatures of the outflow.

Figure 5 shows the 3D $\mathrm{H}_{2}$ emissivity of the outflow projected onto a plane tilted $30^{\circ}$ from the symmetry axis of the outflow. Note that the brightest emission emanates from the dissociation region immediately behind the outer bowshock. Of course the stability properties of the wind collimation mechanism as well as the three dimensional nature of the fragmentation of the thin shell of swept up molecular gas cannot be fully addressed using the 2D cylindrically symmetric approach taken here. Future work will focus on the three dimensional stability of the flow collimation mechanisms as well as on the effect that the outflow geometry has on the fragmentation of protostellar accretion shells, morphology and momentum dissipation efficiency.

\section{Conclusions}

We have presented first results of simulations of molecular outflows using a new Adaptive Mesh Refinement code which tracks both ionzation and chemistry. These simulations focus on the early time evolution of a wide angle wind expanding into collapsing rotating sheet. Our simulations are able to marginally resolve the internal dynamics of the swept-up and with this resolution we find the leading sections of the outflow lobe to be unstable to what appear to be Thin Shell modes. Future work will focus on exploring the dynamics of the outflows in greater detail providing links between the early evolution of the outflow and the late-time large scale appearence. In particular we are interested to see if the fragmentation of the shell changes the global dynamics of 
the outflow in significant ways by generating a "clumpy" lobe which expands and sweeps up ambient material.

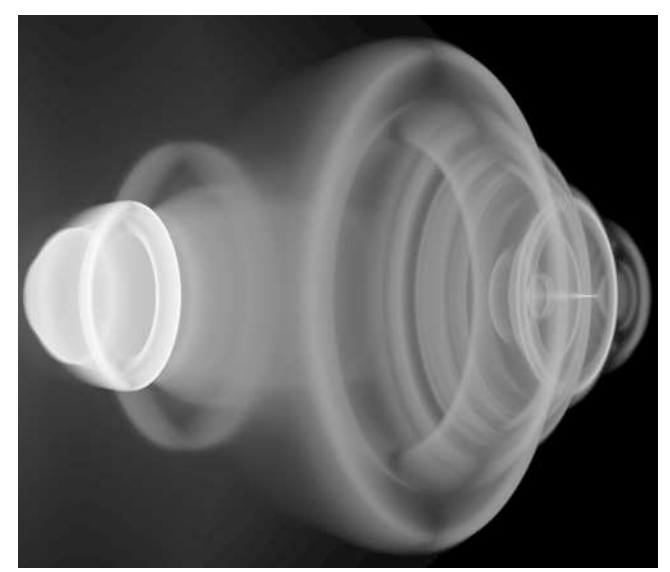

Figure 5. Collapsing sheet outflow, low speed case, synthetic $\mathrm{H}_{2}$ emission projection at $\mathrm{t}=97 \mathrm{yr}$.

\section{References}

Arnaud, M. \& Rothenflug, R. 1985, A\& $A S, 60,425$

Dalgarno, A. \& McCray, R. A. 1972, ARA\& $A, 10,375$

Delamarter, G., Frank, A., \& Hartmann, L. 2000, ApJ, 530, 923

Dove, J. E. \& Mandy, M. E. 1986, ApJL, 311, L93

Hartmann,L., Calvet,N., \& Boss,A. 1996, ApJ, 464, 387

Hollenbach, D. \& McKee, C. F. 1979, ApJS, 41, 555

Hollenbach, D. \& McKee, C. F. 1980, ApJL, 241, L47

Launay, J. M. \& Roueff, E. 1977, A\& A, 56, 289

Lee,C., Stone,J.M., Ostriker,E.C., Mundy,L. 2001, ApJ, 557, 429

Lim, A. J., Raga, A. C., Rawlings, J. M. C., \& Williams, D. A. 2002, MNRAS, 335, 817

Lepp, S. \& Shull, J. M. 1983, ApJ, 270, 578

Mandy, M. E. \& Martin, P. G. 1993, ApJS, 86, 199

Martin, P. G., Keogh, W. J., \& Mandy, M. E. 1998, ApJ, 499, 793

Mazzotta, P., Mazzitelli, G., Colafrancesco, S., \& Vittorio, N. 1998, A\&AS, 133, 403

Poludnenko, A., Varniere, P., Frank, A., \& Mitran S, 2004, to appear in Springer's Lecture Notes in Computational Sciences and Engineering (LNCSE) series

Smith, M. D. 1994, MNRAS, 266, 238

Shu,F.H., Najita,J., Ostriker,E.C., \& Shang,H. 1995, ApJ, 455, 155

Varniere, P., Poludnenko, A., Cunningham, A., Frank, A., \& Mitran S, 2004, to appear in Springer's Lecture Notes in Computational Sciences and Engineering (LNCSE) series

Vishniac, E. T. 1994, ApJ, 428, 186

Vishniac, E. T. \& Ryu, D. 1989, ApJ, 337, 917 